\title{
RSSI Algorithm Based Interference Reduction Technique For LTE Networks
}

\author{
Shahegul Ansari \\ Department of Computer Science \& Engineering \\ G H Raisoni College of Engineering \\ Nagpur, India \\ shahegul.ansari7@gmail.com
}

\author{
Prof. Veena Gulhane \\ Department of Computer Science \& Engineering \\ G H Raisoni College of Engineering \\ Nagpur, India \\ veena.gulhane@raisoni.net
}

\begin{abstract}
Wireless communications networks require 3GPP (Long Term Evolution) technique for gaining highly efficient spectrum and data rates with flexible bandwidth and spectrum allocation. Femtocell is an approach of LTE networks for reducing indoor coverage problems. While, locating macro-cells and femto-cells may found a situation of interference. For reducing this problem, in this paper we propose a Modified Fractional Frequency Reuse (MFFR) technique with Received Signal Strength Indication (RSSI) algorithm. This technique would effectively mitigate the co-channel interference from both interference techniques that is of co tier and cross tier, thus get higher throughput of system.
\end{abstract}

Keywords-Femtocell; Interference Mitigation; MFFR; CoChannel Interference

\section{INTRODUCTION}

To change the traditional communication method of cellular network LTE technology with heterogeneous cellular network is one of the most favorable standards. For improving spectral efficiency and getting high statics rates the Orthogonal Frequency Division Multiple Access (OFDMA) technique is utilized. Orthogonal sequence is used for resource allocation. A technique to remove coverage holes in combined network of small and large cells based co channel deployment is known as heterogeneous cellular networks. In heterogeneous network small cell coverage is used to provide better quality of services and good cell boundary coverage for dissimilar mobile devices. Small cells are nothing but low power nodes such as micro/pico/femto base stations deployed in macro cell [1] [2]. For future improvement the network capacity is very important. And LTE is a good approach for this improvement.

Femtocell is approach of LTE network for reducing indoor coverage problems. Femtocell is a small range, flat power, sealing type user installed home base station toiling on licensed spectrum better utilized by mobile users. Femtocell connection with primary network is via guided medium and cord medium. The femtocell brings various merits to both consumers and operators, such as expanded indoor coverage, increased the system capacity, QoS and reduced wealth and operational cost [4]. Both cells are deployed on same frequency band because of high cost. Because of insufficient macrocell coverage, growth in data services and indoor coverage, the femtocell could be an appealing solution.

The macro and femtocell based interference problem should be resolved, femtocell is basically located under macrocells, and also utilizes the macrocell frequency. Channel devotion is old and simple technique to overcome interference problem, but resource utilization is not proper. Same carrier utilization among various users may cause interference at the cell boundary. The heterogeneous network deployment is based on co-channel [2] [3]. Femtocell is controlled by single user not by the head of network so usually it is on and off or sometime fixed at one location. So, to mitigate co channel interferences problem must have to adjust different operations.

The paper is organized as, In section II describe interference scenarios in heterogeneous based femtocell network, section III, related work, section IV describe the introduction of FFR technique, section $\mathrm{V}$ proposed work and in VI will conclude the paper.

\section{INTERFERENCE SCHEME IN HETEROGENEOUS NETWORKS}

In wireless networks various interference schemes requiring Home Node Base station, macro node base stations, mobile user resources and home user resources. When any node that is restricted with user resources if it is connected with another node base station by downlink then it is downlink interference, if one user resource is getting interfere with another user resource it is known as uplink interference. Figure 1.3.1 and 1.3.2 shows different schemes of cross layer and downlink based interference particularly. Basically, Cotier and Cross-tier are the types of interferences.

- Cross-tier

Macro-Femto Interference

Users of two different cells are working on the same frequency band may cause restriction for others it links macro and femto interference. 


\section{- Co-tier}

Femto-Femto Interference

If one user of femtocell is trying to expand signal may cause interference with other neighbouring femto cells.

The downlink layer based interference framework consists of various causes. The macro cell base station is located as per the femtocell coverage capacity. Another reason is that macro and femto base station are working on the same frequencies also this structure is based on co channel deployment. And main cause is macro base station is able to cover the inner area but in outer area leakage of radiation may cause interference.

Because of glass window and little open space the leakage grows. Because of close group configuration of macrocells interference may occur. Handover of mobile users and macro base station may occur if user gets powerful signal from macro base station.

\section{RELATED WORK}

Generally, the interference is occurs because of two or many more devices while communicating are very near to each other. The wireless networks faces lot many interference issues. For example, when the same channel is utilized by two different users then interference occurs which is Co-channel interference (CCI). Co-tier and cross-tier interferences come further when mobile station send data on the channel which is already in use by near user. Heterogeneous Cellular Networks are high density networks that will have co-tier and cross-tier interference because of high and advance technology.

Frequency and spectrum partitioning based ALOHA (F-ALOHA) [5] strategy were applied, it use to discard interference among femtocells and macrocells by assigning OFDMA spectrum. For sub channels utilization all the femtocells in the network are works in arbitrary manner. Femtocell can resolute an optimal spectrum portion. Though, this is a devoted frequency based approach. In this technique the whole bands are not worked directly with macrocells if the spectrum utilization is adaptive.

Dynamic Frequency Planning (DFP) [6] was one of another technique for reduction of interference. When same frequency transmission is done in the sector the sector calculation is necessary. Femtocell which is organized by the self-owner does not have optimization function but here to reduce the overall network system optimization function is run.

There are various algorithm available for frequency allocation one of them is Least Interference Power (LIP) [7] algorithm is suggested for reducing interference to selects the frequency section one powered-up femto base station was established. In this algorithm frequency assignment is based on femtocell on and off time management. So femtocell location is not arbitrary manner but it is in sequential manner. Estimation of interference between femtocells and macrocells are not possible.
Private and coupled model technique [8] was proposed for user location which was not able to perform by OFDMA techniques under femtocells, in this technique for resource allocation femto and macro users are divides based on time and frequencies. Femtocell reused the cell boundary based macro users resources in coupled replica on the other side the femtocells located on centre area reused the orthogonal macrocell based resources. So for OFDMA structure no other FFR technique is utilized by this scheme.

FFR-3 (Fractional Frequency Reuse with 3 frequency sub bands) [9][10] suggested as femto macro network suited technique. This strategy splits the cell into three parts with adding one middle region to utilize the user's diversity among macro and femtocell. Where macrocells used the fractional frequency reuse scheme by dividing into three parts and femtocells are allotted with orthogonal sub-bands varying from macro users in one cell. This strategy gives positive result in reducing cross tier interference, but sometimes it may happen at the border linked cell.

Spectrum Swapping [11] [12] is one of the technique used for the upgrading the performance of macrocell and solving the near and far problems that typically occur. Orthogonal frequency band is used for frequency carrier allocation and divided into three parts. But received SINR resulted that an outer coverage problem will occurs with high probability because of near far problem. In one macrocell number of femtocell are able to create and each and every femto cell have different frequency sub bands from macrocell. When macrocell and femtocell orthogonally handle by each other than interference reduction between them must be performed. Received signal are best to joints the femtocells and also upgrade the performances of global and local networks. It requires higher complexity.

The Optimal frequency partitioning [13] [14] that maximize the frequency sum-capacity and split frequencies into macro devoted shared and femto devoted resources. A fractional spectrum splitting scheme for only femto-cell edge area and establish a resource allocations optimization problem to maximize the system rate. But it decline spatial reuse and spectral efficiency.

For co channel interference reduction pilot sensing [15] technique was proposed. The FRF strategies are assigned to macrocells, and remaining frequency sub bands are used by the femtocells. Consider here, three reuse elements factors for macro cell, from three if I is used by the macrocell and II and III are selected by femtocell. So the system capacity is increased and the throughput of macrocell is minimizes. The macrocell based reuse elements conflicts the other LTE and Wi-MAX network where only one is able to reuse element. For cell boundary interference reduction best option is FFR based on macrocell structure. At this state in network, as per femtocell evolution the macro user interference must be reduced. So the focus is on FFR based interference reduction technique. 
TABLE I COMPARISON AMONG METHODS OF INTERFERENCE REDUCTION BASED ON PARAMETERS

\begin{tabular}{|l|c|c|}
\hline \multicolumn{1}{|c|}{ Schemes } & Throughput & SINR \\
\hline F-ALOHA[5] & 17 & $5 \mathrm{~dB}$ \\
\hline DFP [6] & 16 & $5.5 \mathrm{~dB}$ \\
\hline LIP [7] & 16.5 & $6 \mathrm{~dB}$ \\
\hline PCM [8] & 15 & $7.9 \mathrm{~dB}$ \\
\hline FFR-3 [9][10] & 18.0 & $9 \mathrm{~dB}$ \\
\hline
\end{tabular}

The above table shows the comparison among various methods of interference reduction based on parameters like SINR, throughput. The methods described above are divided as FFR based and without FFR method. For the above given techniques SINR is calculated as $9.5 \mathrm{~dB}$ for the FFR based technique and for NoFFR the value is 0.60 . The throughput is based on the size of femto users if the users are increasing then throughput get decrease if user is less than throughput is more [5] [6] [7] [8]. When the femtocell is 300 then for without FFR technique throughput is $16.5 \mathrm{Mbps}$, similarly for the FFR based technique the throughput is $18.0 \mathrm{Mbps}$.

In beginning works they utilized FFR based and NoFFR techniques. For the NoFFR based technique only one frequency band is allocated which result in high interference. Another side for the FFR technique 3 frequency bands are allocated but divided into another sub bands. The macro and femto users utilize these sub bands. This method provide better results than without FFR technique. Interference issue between femto and macro cells reduced here but the co channel problem remains constant. This will be reducing by our proposed method as described below with the help of utilizing the more frequency sub-bands and also improving the SINR, throughput ratio.

\section{FRACTIONAL FREQUENCY REUSE TECHNIQUE}

The frequency organization is a superb option to reduce adjacent macrocell based interference; it is an uneconomical employment based on system bandwidth capacity. Frequency spectrum is very expensive resource, so it must be optimally utilized. Because of huge number of wireless devices increasing the frequency spectrum is becoming rare resource. Some new techniques are used to utilize the spectrum carefully so that today's bandwidth requirement is able to fulfill. For bandwidth enhancement and proper consumption over the network easy and useful method is FFR technique [16].

The basic concept of FFR technique is to divide the frequency band so that adjacent cells does not try to interfere with each other, so that adjacent cell based interference will reduced, though utilizing whole spectrum band as compare to the conventional frequency method [16] [17]. This method divides the one single cell into two parts; so that one part is at the center area means near to the base station and other is at the boundary region. Like this total considered band of frequency is divided into different parts as sub bands and some are assigned for the inner area and some are for the outer area.

Two types of FFR is available; one is Hard FFR and other is Soft FFR. For hard FFR scheme combine cells use the definite frequency while at the boundary area only unused frequency bands are utilized. Soft FFR also work as similar to hard FFR but advantage of soft is it utilize bandwidth better than hard FFR, but have some disadvantage inter-cell interference scenario occurs more than hard FFR [18].

\section{PROPOSED WORK}

Basic focus of paper is mitigating the interference, for this purpose four sub bands are used by the network. Cell is divided into two parts, center area and boundary area. This work is able to provide more number of frequency bands to the femtocells which are located at the boundary area. With the help of this interference at the boundary area are able to reduce and so the throughput will increased. So the performance will better.

The macro and femto cell based frequency sub bands are differentiated as shown in the below figure 5.1. Middle and boundary area are the two components of macrocell coverage, three sectors are allocated for each cell, these sectors and areas are indicated by A1, A2 A3 and S1, S2, S3. One frequency band is spitted into components, and each component is classified into three parts, which are signified as P, Q, R and S. For macrocell, different sub bands are assigned to the macrocell areas to use the modified fractional frequency reuse (MFFR). The reuse element is registered for middle region, and the other three elements are assigned to boundary region. The $\mathrm{P}$ is used by the center area for $\mathrm{A} 1, \mathrm{~A} 2$ and $\mathrm{A} 3$ and sub bands $\mathrm{Q}, \mathrm{R}, \mathrm{S}$ are registered in the different sectors $\mathrm{S} 1, \mathrm{~S} 2$ and $\mathrm{S} 3$ respectively.

Femtocell utilized all the sub bands which are not used by the macro users for the above considered situation. Especially, when the femtocell is located at the middle area, this does not allow the sub bands in the edge sector S1 which is already utilized by the macrocell, it utilizes sub-bands $\mathrm{P}, \mathrm{Q}$, or S, while sub-band Q employs for the macrocell. For area A1 based femtocells the $\mathrm{R}$ and $\mathrm{S}$ sub bands are already registered. The sub band $\mathrm{P}$ is avoided by the femtocell because it is utilized by the macrocell for sector S1. It also avoids the sub band Q, which is already utilized by the macrocell. The Q sub band received the contrast signal strength power as shown in sector $\mathrm{S} 1$.

The OFDMA technique causes the macrocell interfered with inter cells, this interference are going to reduce by the proposed Modified Fractional Frequency (MFFR). For interference avoidance among femto and macro users the femtocells utilize the different sub bands. As per the accessibility macrocell utilized the sub bands, because transmission power of femtocell is low. Because of this the interference among femto and macro cells avoidance is significant. For the superior output of boundary users, more number of sub carriers need to be allotted to the inside boundaries. 

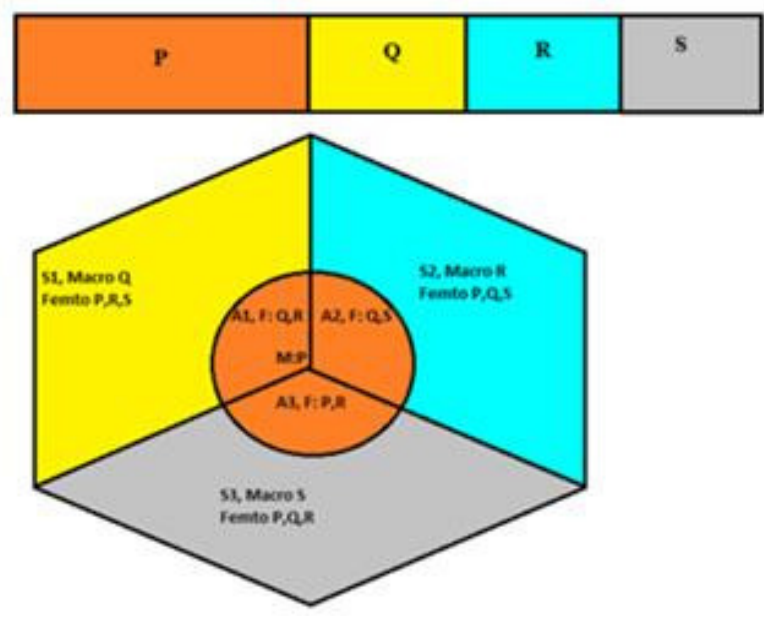

Fig: Proposed Interference Management scheme using FFR

\section{A. Proposed Algorithm}

As shown in the figure above according to the FFRE technique the users of macro cells are splitting among sub carriers of sub bands. Spitted frequency sub bands are used by the center and boundary areas of the cells.

Macrocell signal is identified by the adjacent femtocells when network is working. The received signal strength indication algorithm is used for finding signal strength of all the sub bands $\mathrm{P}, \mathrm{Q}, \mathrm{R}$ and $\mathrm{S}$. At the center area if the signal strength of the $\mathrm{P}$ sub band is powerful then based on signal strength the femtocell is detected in the center. If the Q sub band is having strong signal, then at the area A1 femtocell is detected. The sub carriers selection are depend on the sub bands $\mathrm{R}$ and $\mathrm{S}$. The strong signal power are used for the macro users, the sub band $\mathrm{P}$ and $\mathrm{Q}$ are prohibits. This is similar for $\mathrm{A} 2$ and $\mathrm{A} 3$.

Another side, boundary area is based on the femtocell, if the signal strength of $\mathrm{P}$ area is poor. If $\mathrm{Q}$ sub bands have strong signal then in area A1the femtocell is placed. From sub bands $\mathrm{P}, \mathrm{Q}$, and $\mathrm{S}$ sub carriers are selected for femtocell. Based on the signal strength $\mathrm{J}$ sub band is selected.

\section{Algorithm for Localization}

\section{Checking Received Signal}

If (a sending node is already in locating table \&\& sending node is at the bottom)

Calculate delay time (Pr-RSSI) Wait for as much as delay time

$$
\}
$$

If (a node receive didn't receive signal from other node) When finishing waiting time $\& \&$ node is not yet found

OR

If is back anchor node

\section{\{}

Node which has shortest delay time will broadcast RSSI (Strongest=Node-ID)

\}

Else

Other nodes save the RSSI info to its own location table;

For strongest node

Strongest_Node .Is it bottom = true;

Stongest_Node. $X$ axis $=$ Sending_Node.X;

Strongest-Node. $\mathrm{Y}$ axis $=$ Sending_Node. $\mathrm{Y}-1$;

Strongest-Node. $Z$ axis $=$ Sending_Node.Z;

Sending_Node $=$ Strongest_Node;

\section{CONCLUSION}

Femtocell technology is an original investment for the user and subscriber, since it allows low cost and better spectral usage and Quality of service. This paper is basically focused on the macrocell and femtocell based interference reduction. Different technologies were discussed in this paper, based on their results we concluded that they are not able to provide better service at cell edge region. Hence, we proposed an efficient and simple MFFR with RSSI algorithm based interference reduction technique in the LTE femtocell systems. The macrocell coverage is spitted into boundary and center region with three sectors for each parts.

\section{REFERENCES}

[1] Amitabh Ghosh, Jeffery G. Andrews, et al,. "Heterogeneous Celular Networks: From Theory to Practice", IEEE Communication Magazine, June 201.

[2] Alecksandar Damanjanovic et al., "A Survey on 3GPP Heterogeneous Networks", IEEE Wireless Communication, June 2011.

[3] Heli Zhang, Shanzhi Chen, Xi Li, Hong Ji, And Xiaojiang Du, "Interference Management for Heterogeneous Networks with Spectral Efficiency Improvement”, IEEE Magazine Wireless Communication April 2015.

[4] Mayur C Akewar, Dr Nieshsingh V. Thakur, "A Study of Wireless Mobile Sencor Networks Deployment", IRACST-International Journal of Computer Networks \& Wireless Communications (IJCNWC), ISSN2250-3501, Vol 2, No 4, August 2012.

[5] V. Chandrasekhar, J. Andrews, "Spectrum Allocation in Two-Tier Networks", IEEE Asilomar Conference on Signal System and Computers, Oct. 2008.

[6] D. Lopez-Perez et al., "Interference Avoidance and Dynamic Frequency Planning for WiMax Femtocells Networks", IEEE International Conference on Communication Systems (ICCS), Nov. 2008.

[7] H. Zeng, C. Zhu, and W. Chen, "System Performance of Self Organizing Network Algorithm in WiMax Femtocells", ACM International Conference on Wireless Internet (WICON), Nov. 2008.

[8] K. Sundaresan, S. Rangarajan, "Efficient Resource Management in OFDMA Femto Cells", ACM International Symposium on Mobile Ad Hoc Networking and Computing (MobiHoc), May 2009.

[9] Esfahani, M.N \& Ghahfarokhi, B. S. "Improving Spectral Efficiency in Fractional Allocation of Radio Resources to Self Organized Femtocells 
Using Learning Automata", In Telecommunications (IST), $20147^{\text {th }}$ International Symposium on, September 2014, pp. 1071-1076.

[10] Fradi, N. Najeh , S. \& Boujemaa, H. "Resource Allocation in OFDMA Networks with Femto and Macrocells Coexistence using Fractional Frequency Reuse (FFR)", In Communications and Networks (ComNet), 2014 International Conference on, March 2014, pp. 1-5.

[11] Jin, F. Zhang, R. \& Hanzo, L. "Fractional Frequency Reuse Aided Twin Layer Femtocell Networks: Analysis Design and Optimization", Communications, IEEE Transactions on, 61(5), 2013, pp. 2074-2085.

[12] Benkhelifa, F., Rezki, Z., \& Alouini, M.S. "Cooperative Decoding in Femtocell Networks: Performance Complexity tradeoffs" In Signal Processing Advances in Wireless Communications (SPAWC), 2012 IEEE $13^{\text {th }}$ International Workshop on June 2012, pp. 404-408.

[13] Jeon, W. S., Kim, J., \& Jeong, D. G. "Downlink Radio Resource Partitioning with Fractional Frequency Reuse in Femtocell Networks", Vehicular Technology, IEEE Transactions on, 63(1), 2014, pp. 308-321.
[14] Tran G K, Tajima S, Ramamonjison R, et al. "Study on Resource Optimization for Heterogeneous Networks[J]", Ieice Transactions on Communications, 2012, 95(4), pp. 1198-1207.

[15] T. Kim, T. Lee, "Throughput Enhancement of Macro and Femto Networks By Fractional Frequency Reuse and Pilot Sensing," IEEE International Performance, Computing and Communications Conference (IPCCC), Dec. 2008.

[16] Mustafa Y. Arslan, Jongwon Yoon, et al. "A Resource Management System for Interference Mitigation in Enterprise OFDMA Femtocells", IEEE Transaction on Networking, Vol. 21, No.5, October 2013.

[17] Husam Eldin Elmustasim Osman Mohamed Elfadil, et al. "Fractional Frequency Reuse in LTE Netwokrs”, IEEE Magazine 2015.

[18] Dimitrios Bilios, et al. "Optimization of Fractional Frequency Reuse in Long Term Evolution Networks", IEEE Wireless Communications and Networking Conference; Mobile and Wireless Networks, 2012. 